\title{
Screening subclinical keratoconus with Placido-based corneal indices
}

D. Ramos-López, A. Martínez-Finkelshtein, G. M. Castro-Luna, N. Burguera-Gimenez, A. Vega-Estrada, D.P. Piñero and J. L. Alió

Optometry \& Vision Science

2013

This is not the published version of the paper, but a pre-print.

Please follow the link below for the final version and cite this paper as:

D. Ramos-López, A. Martínez-Finkelshtein, G. M. Castro-Luna, N. Burguera-Gimenez, A. Vega-Estrada, D.P. Piñero and J. L. Alió. Screening subclinical keratoconus with Placido-based corneal indices. Optometry \& Vision Science, Volume 90 (4), Pages 335-343, ISSN 1040-5488 (2013).

http://dx.doi.org/10.1097/OPX.0b013e3182843f2a 


\section{Screening subclinical keratoconus with Placido-based corneal indices}

Darío Ramos-López, MSc

Department of Statistics and Applied Mathematics, University of Almería, Spain

Andrei Martínez-Finkelshtein, $\mathrm{PhD}$

Department of Statistics and Applied Mathematics, University of Almería, Spain and Institute Carlos I of Theoretical and Computational Physics, Granada University, Spain.

Gracia M. Castro-Luna, MD, PhD

VISSUM Corporation, Almería, Spain

Neus Burguera-Gimenez, MSc

Keratoconus Unit. VISSUM Corporation, Alicante, Spain

Division of Ophthalmology, Universidad Miguel Hernández, Alicante, Spain

Alfredo Vega-Estrada, MD, MSc

Keratoconus Unit. VISSUM Corporation, Alicante, Spain

Division of Ophthalmology, Universidad Miguel Hernández, Alicante, Spain

David Piñero, PhD

Departamento de Óptica, Farmacología y Anatomía, Universidad de Alicante, Spain

Jorge L. Alió, MD, PhD

Keratoconus Unit. VISSUM Corporation, Alicante, Spain

Division of Ophthalmology, Universidad Miguel Hernández, Alicante, Spain 
Address all correspondence to:

Andrei Martinez-Finkelshtein,

Department of Statistics and Applied Mathematics,

University of Almería, 04120 Almería, Spain;

Fax: +34950015167, e-mail: andrei@ual.es

This paper contains 6 Tables and 3 Figures.

Submitted on September 19, 2012 


\section{Abstract}

\section{Purpose:}

To assess in a sample of normal, keratoconic and keratoconus suspect eyes the performance of a set of new topographic indices computed directly from the digitized images of the Placido rings.

\section{Methods:}

This comparative study comprised a total of 124 eyes of 106 patients from the ophthalmic clinics Vissum Alicante and Vissum Almería (Spain), in three groups: control group (50 eyes), keratoconus group (50 eyes) and keratoconus suspect group (24 eyes). In all cases, a comprehensive examination was performed including the corneal topography with a Placido-based CSO topography system. Clinical outcomes were compared among groups, along with the discriminating performance of the proposed irregularity indices.

\section{Results:}

Significant differences at level 0.05 were found on the values of the indices among groups by means of Mann-Witney-Wilcoxon non-parametric test and Fisher's exact test. Additional statistical methods, such as receiver operating characteristic analysis and $\mathrm{K}$ fold cross-validation, confirmed the capability of the indices to discriminate between the three groups.

\section{Conclusions:}

Direct analysis of the digitized images of the Placido mires projected on the cornea is a valid and effective tool for detection of corneal irregularities. Although based only on the data from the anterior surface of the cornea, the new indices performed well even when applied to the keratoconus suspect eyes. They have the advantage of simplicity of calculation combined with high sensitivity in corneal irregularity detection, and thus can 
be used as supplementary criteria for diagnosing and grading keratoconus that can be added to the current keratometric classifications.

Keywords: Corneal irregularities; subclinical keratoconus; irregularity index; diagnosis; corneal topography; Placido disks 
Keratoconus (KC) is an ectatic debilitating corneal disorder characterized by a progressive corneal thinning that results in corneal protrusion, irregular astigmatism, and decreased vision ${ }^{1}$. Corneal elasticity and rigidity is severely affected in keratoconic eyes ${ }^{2-4}$, which become more susceptible to the effect of any pressure, such as the intraocular pressure. Consequently, the corneal shape is more easily distorted (corneal steepening and aberrometric increase in KC). This explains the usual significant increase in the anterior corneal irregularity and a deterioration of the visual quality in $\mathrm{KC}$, aggravated by the high optical relevance of the first surface of the cornea.

Several grading systems have been described in the literature in order to classify the severity of $\mathrm{KC}^{5-7}$. Most of these grading systems have been developed taking into account the visual performance of the patient, topographic morphology of the disease, the corneal keratometry readings and corneal aberrometry ${ }^{8-10}$, and have been proven to be an essential tool in the therapeutic approach to the management of $\mathrm{KC}$.

Nevertheless, there is a form of this disease, characterized by a milder modification in corneal topography and morphology but without the impairment of the visual function of the patient, that has been defined as an early $\mathrm{KC}$, subclinical $\mathrm{KC}$, or $\mathrm{KC}$ suspect. One of the main difficulties in relation to this entity is the lack of its clear definition in the literature ${ }^{11}$.

The topographic analysis of the anterior corneal surface is the main tool that has been used for the KC diagnosis and characterization for years. Several indices, both simple and compound, decision trees and even neural networks based on the corneal topographic data and optical parameters have been developed to provide a more reliable tool to detect abnormal and borderline suspect corneas ${ }^{12-26}$. Also the vertical coma of the corneal aberration is one of the simplest direct KC markers used in the clinical practice ${ }^{9,13}$. However, and even with the advance of the technological tools employed today for the assessment of potential candidates for refractive surgery, subclinical $\mathrm{KC}$ is still considered the most important risk factor for 
developing post LASIK ectasia ${ }^{27-28}$, a devastating condition leading to a significant visual impairment of the patient. Thus, improving the screening strategies, tools and techniques that allow us to identify those cases with the potential hazard of developing such a feared complication has become a major challenge within the ophthalmic community.

Most of the corneal indices, published in the literature, are based on the elevation or curvature data of the cornea, as well as pachymetry ${ }^{29}$ or the epithelial thickness profile ${ }^{30}$. However, and at least in the case of the dominant Placido-based topographers, these data are not obtained by a direct (and verifiable) measurements, but are an outcome of a mathematical processing of the image of the rings in the keratographic picture by more or less sophisticated (and in the case of commercial devices, by proprietary and not always transparent) algorithms ${ }^{31-33}$. These procedures make important assumptions on the corneal shape (rotational symmetry, approximability by cubic splines, etc.) that are difficult to satisfy in the case of a very complicated or irregular corneal surface. Therefore, numerical approaches developed for $\mathrm{KC}$ detection from topographic data using these reconstruction algorithms inherit unnecessarily the complexity of the currently used ring image-to-curvature conversion methods, as well as might be affected by the unavoidable intrinsic errors appearing during such a conversion ${ }^{34-35}$.

In order to overcome these shortcomings, as well as to improve and complement the existing set of corneal disease markers, a set of new irregularity indices has been introduced recently ${ }^{36}$. These indices bypass the conversion to corneal power and use directly the digitized image of the Placido rings.

The previous contribution ${ }^{36}$ had a methodological character, although some preliminary discussion of the performance of the indices was carried out there. The aim of this current study is to assess in a sample of normal, keratoconic and keratoconic suspect eyes a 
simplified subset of the topographic indices proposed in that paper, evaluating their potential as a tool for $\mathrm{KC}$ detection.

As a final remark, we should point out that any additional information about a cornea, such as its pachymetry, could improve considerably the screening capability of any marker. The indices analysed here use only the data available to a Placido-based topographer (which are still a vast majority in the clinical practice), but we hope they help to use these data more efficiently.

\section{Methods}

This case series comparative study comprised a total of 124 eyes of 106 patients. Two Spanish ophthalmologic centers participated in the recruitment of patients for this study, Vissum Alicante and Vissum Almería, forming part of the Thematic Network of the Cooperative Sanitary Research (RETIC) RD07/0062. All these cases were assigned to one of the following three groups depending on the presence or not of $\mathrm{KC}$ : a control group, which included 50 eyes (from 50 patients), a KC group, which included a total of 50 eyes (from 32 patients), and a subclinical $\mathrm{KC}$ or $\mathrm{KC}$ suspect group, with a total of 24 eyes (from 24 patients).

The inclusion in the $\mathrm{KC}$ group was based on the standard criteria for the diagnosis of this corneal condition and the absence of any previous surgical intervention that could have altered the corneal properties. The following signs were considered at diagnosis ${ }^{1}$ : corneal topography revealing an asymmetric bowtie pattern with or without skewed axes and at least one keratoconus sign on slit-lamp examination, such as stromal thinning, conical protrusion of the cornea at the apex, Fleischer ring, Vogt striae or anterior stromal scar. In those patients wearing contact lenses for the correction of the refractive error, only data obtained after an appropriate contact lens discontinuation were considered: at least 2 weeks for soft contact lenses and at least 4 weeks for rigid gas permeable contact lenses. The exclusion criteria for 
the $\mathrm{KC}$ group were other ocular active pathology at the moment of diagnosis and the presence of an advanced KC (grade 4 according to the Alió-Shabayek grading system ${ }^{8}$ ). In cases of unilateral $\mathrm{KC}$, the affected eye was always included in the study. However, in bilateral $\mathrm{KC}$ only one eye was selected randomly for the study.

The group of normal eyes or control group only included eyes with no other ocular pathology, previous ocular surgery or irregular corneal pattern. In this control group, only one eye from each patient was selected randomly (random sampling) for the inclusion in the study in order to avoid the potential bias introduced by the correlation between both eyes of a same patient.

The definition of $\mathrm{KC}$ suspect cases was based on the following clinical and topographic evaluation: no slit-lamp findings, no scissoring on retinoscopy, and the presence of asymmetric bowtie (AB), inferior steepening (IS), skewed axes (SRAX) or asymmetric bowtie with skewed axes (AB/SRAX) pattern on topography ${ }^{10}$.

All patients were informed about the study and signed an informed consent document in accordance with the Helsinki Declaration.

\section{Examination protocol}

The corneal topographic analysis was carried out with the CSO topography system (CSO, Firenze, Italy). This topographer analyses a total of 6144 points of a corneal area enclosed in a circular annulus defined by an inner radius of 0.33 and an outer radius of $10 \mathrm{~mm}$ with respect to the corneal vertex. The software of this system, the EyeTop2005 (CSO, Firenze, Italy), performs automatically the conversion of the corneal elevation profile into corneal wavefront data using the Zernike polynomials with an expansion up to the $7^{\text {th }}$ order, although it allows to export the raw data (positions of the digitized mires) as an ASCII file. For the sake of reliability of the analysis of the indices, the standard KPI index as well as the 
I-S index has been stored for comparative purposes. Both indices are well known and precisely defined in the literature ${ }^{7,33}$.

\section{Definitions of the corneal indices}

It is convenient to point out that in the description of the indices we skip the initial discretization step, performed by every commercially available topographer using presumably standard and widely available edge-detection procedures, when the high-contrast black-andwhite images of the mires are converted into a discrete points set. Hence, we assume as the input data the coordinates of these points along the edges of consecutive mires, which we consider as positions of the digitized mires. With this information, we have calculated the irregularity indices following the previously discussed methodology ${ }^{36}$. From the original set of indices, we used a small subset of the best performing indices (also the most robust ones with respect to the misalignment of the eye and other errors), complemented with an additional index as described below.

The digitized points $P_{j}$ captured by the camera of the Placido disk corneal topographer were grouped in $N$ 15mires. For the sake of precision, we assume that there were 256 points equally spaced along each ring corresponding to the same number of semimeridians (a value found in a majority of existing devices). We used only data from complete rings, limiting the number of rings to the maximum of 15 . The indices were defined according to the information obtained from all mires as follows.

For each $k$, the center $C_{k}$ and radius $R_{k}$ of the best-fit circle for the $k$-th mire was calculated using a standard least squares procedure ${ }^{37}$, along with the following primary indices $(P I)$ :

$-P I_{1}$ : the diameter of the set of centers $\mathrm{C}_{\mathrm{k}}$ (normalized by the total number of rings $\mathrm{N}$ ) 


$$
P I_{1}=\frac{1}{N} \max _{1 \leq n, m \leq N}\left\|C_{n}-C_{m}\right\|
$$

$-P I_{2}:$ the total drift or the deviation in the consecutive centers $C_{k}$ :

These two indices give global information about the deviation of the image of the

$$
P I_{2}=\frac{1}{N-1} \sum_{1 \leq n \leq N-1}\left\|C_{n+1}-C_{n}\right\|
$$
rings from a concentric pattern.

Data from mires were also fit with an ellipse with the aim of capturing the spatial orientation and deformation of each mire (see Figure 1) by means of a simplification ${ }^{37-41}$ of efficient methods for computation of the best-fit ellipse, rendering the following asymmetry index:

$-P I_{3}$ : the dispersion of the values of the axis ratios $r_{k}=a_{k} / b_{k} \geq 1$ of the $k$-th best fit ellipse by means of the following expressions:

$$
P I_{3}=\sqrt{\frac{1}{N} \sum_{1 \leq k \leq N}\left(r_{k}-\bar{r}\right)^{2}} \quad \text { where } \quad \bar{r}=\frac{1}{N} \sum_{1 \leq k \leq N} r_{k}
$$

Indices $P I_{1}-P_{3}$ coincide with those defined previously ${ }^{36}$. They were complemented by some additional indices whose definition was modified with respect to that given previously ${ }^{36}$, seeking better discrimination ability and robustness. In particular, we avoid the use of polar coordinates (sensitive to the apex misalignment), calculating the indices $A R(k)$ from the original image of the mires as the radius of the best-fit circle to the $k$-th ring. In practice, only the fourth mire (index $A R(4)$ ) was used in the combined model described below, and thus only its individual performance will be analyzed in the next section.

We also carried out the standard linear regression of the coordinates of the centers $C_{k}=\left(x_{k}, y_{k}\right)$, yielding the coefficients for the linear fit $y=a x+b$. With this approach, high values of $a$ correspond to a vertical alignment of the centers, so its value contains information 
about their spatial distribution (see Figure 2). These considerations motivate the following index (we use the name of an index defined previously ${ }^{36}$, but with a new meaning):

$-P I_{4}$ : is the absolute value of the slope of the linear regression,

$$
P I_{4}=|a|
$$

Each of these metrics can be used for $\mathrm{KC}$ detection (or at least, as a measure of corneal irregularity), but as it usually happens with the individual indices, none achieves the necessary sensitivity and specificity to meet the standards. For this reason, a combination was used to improve the detection efficiency. We added to our protocol of indices a new additional combined metric called GLPI, which takes continuous values between 0 and 100 ( $0 \%$ corresponding to a totally normal, and $100 \%$, to a totally altered cornea).

GLPI: is a generalized linear (Placido-based) model combining four of the individual indices mentioned above. Their linear combination (with fixed coefficients) is evaluated in the so-called "probit" link function ${ }^{42-43}$. This yields a quantity between 0 and 1 that is multiplied by 100 for convenience. This value, in the interval $[0,100]$, is a $\%$ of irregularity of the cornea. This definition of GLPI is slightly different from the one given previously ${ }^{36}$ : it has been modified to achieve a better accuracy with a smaller number of individual indices and also to include the redefined index $\mathrm{PI}_{4}$ :

$$
\begin{gathered}
G L P I=100 \times \operatorname{Probit}(\eta), \quad \text { with } \\
\eta=10^{-2}\left(15.7+1043.9 \times P I_{1}-184.2 \times P I_{3}-30.0 \times A R(4)+0.5 \times P I_{4}\right)
\end{gathered}
$$

Statistical analysis

In order to determine the homogeneity of the sample, when divided into training and test sets, a Mann-Witney-Wilcoxon non-parametric test ${ }^{44-45}$ was applied to each of the primary indices. Without assumption of normality, this test checks whether the two samples 
come from the same population (null hypothesis). It can also be used to analyze the discriminating ability of the indices, checking if it renders different values in each group.

Additionally, Fisher's exact test ${ }^{46-47}$ is a statistical method used when a dichotomous classification process is made. This test checks whether the classifier has enough discrimination ability, and it is valid for any sample size. The idea is to compare the expected proportions of false/true positives/negatives with the actual proportion obtained after classifying. This procedure has been used in this study to check if the true proportions of success of the primary indices when classifying normal and keratoconic eyes are independent and consequently, if the primary indices show classification ability or not.

The K-fold cross-validation is a standard statistical tool to assess the global accuracy of a regression or classification model ${ }^{48-49}$. The main benefit of this method is that it makes use (independently) of the same data to fit the model and to check its performance, which is useful when the sample size is relatively small. The sample is divided into $\mathrm{K}$ groups of approximately equal size. Then the regression model is fit (or re-fit, if an initial model was specified) to the data using $\mathrm{K}-1$ of the $\mathrm{K}$ subsets, and its accuracy is measured with the predicted values for the remaining group. When $\mathrm{K}$ becomes equal to the sample size, this scheme reduces to the well-known leave-one-out cross-validation method. This technique allows estimating the global accuracy of a classification method with only one dataset, but using independently subsets of the sample to fit and to validate the model.

Finally, the receiver operating characteristic (ROC) curve analysis is a wellestablished tool for assessing the discriminating capability of a model. We present the results of this analysis for the redefined primary indices $\mathrm{PI}_{4}$ and $\mathrm{AR}(4)$. The ROC curves for the rest of the indices can be found in the literature ${ }^{36}$. 
The primary indices have been computed for all three groups in the database and their means and standard deviations were calculated (see Table 1). The classification ability of the primary indices was assessed in different ways. First, according to the Mann-WitneyWilcoxon tests, most of the indices are able to discriminate between the three groups (see Table 2), except for $\mathrm{PI}_{2}$ and $\mathrm{AR}(4)$, which being appropriate for discrimination between keratoconic eyes $(\mathrm{KC})$ and the rest of the eyes, do not perform well discriminating between normal $(\mathrm{N})$ and keratoconus suspect (KS) eyes. In addition, Fisher's test for all these indices indicated that the true proportions of positives within the $\mathrm{N}$ and $\mathrm{KC}$ groups differ (with a significance level of 0.05 ), so they actually have sensitivity to detect irregularities. Moreover, the ROC curves for $\mathrm{PI}_{4}$ and $\mathrm{AR}(4)$ illustrate the discrimination ability of these indices (see Figure 3); the values of $\mathrm{A}_{\mathrm{z}} \mathrm{ROC}$ (area under the ROC curve) for all the indices appear on Table 2.

Concerning the combined indices, GLPI index computed using the whole database was able to reach the accuracy value 1 (perfect classifying capability between $\mathrm{N}$ and $\mathrm{KC}$ groups). The estimations rendered by the K-fold cross-validation method for different values of $\mathrm{K}$ are shown in Table 4, exhibiting consistent accuracy values between 0.94 and 0.95 .

It is well known that the vertical coma (computed as the absolute value of the Zernike coefficient $\mathrm{Z}_{3}^{-1}$ ) is a simple marker for detecting $\mathrm{KC}^{9,13}$. It is actually very close in spirit to our irregularity index $\mathrm{PI}_{4}$ both measure the upper-down asymmetry, although $\mathrm{PI}_{4}$ follows the ideology of using only straightforward calculations from the mire images. For comparative reasons, the vertical coma has been also computed for all three groups in our database. According to a previous analysis ${ }^{36}$, a suitable cut-off value for the vertical coma to discriminate between keratoconus and normal eyes is $3.59 \times 10^{-5}$. With this threshold, $8 \%$ of the eyes in the $\mathrm{KC}$ group of our database were classified as regular and $4 \%$ of normal eyes 
were classified as irregular, which is a good performance. However, within the keratoconus suspect group (KS), the vertical coma was able to classify only $29 \%$ of those corneas as irregular. To achieve a success rate of 0.79 within this group (the same as $\mathrm{PI}_{4}$, see Table 6), the cut-off value has to be set approximately to $2.00 \times 10^{-5}$, yielding that $22 \%$ of normal eyes are classified as irregular. This is a much lower accuracy in comparison with $\mathrm{PI}_{4}$.

There is a clear similarity in the philosophy of the construction of the KPI and the GLPI indices: both are compound indices, indicating a degree of certainty of detection of a corneal irregularity, with moderate to severe cones receiving a KPI score of $100 \%{ }^{7,50-51}$. Both indices are derived by a variation of discriminant analysis applied to a control group of patients, although GPLI, unlike the KPI, uses only the primary information provided by the keratoscope.

A comparison of the new indices with the KPI and I-S renders some interesting conclusions. For the keratoconus suspect group (KS) their values are summarized in Table 5. For the KPI, we used the standard cut-off reported in the literature, considering values equal to or greater than 23 as anomalous (the first two rows in Table 5 fall within the KPI range for normal eyes, while the last two rows correspond to anomalous ones); in the case of the I-S index, values equal or greater than 1.5 were considered anomalous (now, the first two columns in Table 5 correspond to normal eyes, according to the I-S index, and the last two columns correspond to anomalous eyes). It follows from Table 5 that KPI was able to detect only 6 out of 24 keratoconus suspect eyes (25\%), while I-S was able to detect 12 out of 24 (50\%); moreover, 8 out of 24 cases were not detected by either indices (33.3\%), and only 4 out of 24 cases are detected by both indices simultaneously (16.7\%).

Finally, Table 6 shows that the classification power of GLPI and KPI are very similar in all three groups: normal eyes, keratoconus eyes and keratoconus suspect eyes. Index $\mathrm{PI}_{4}$, exhibiting a reasonable behavior within the group of normal eyes, has a slightly lower $\mathrm{KC}$ 
detection capability than either GLPI or KPI. However, within the crucial group of KS eyes, both GLPI (accuracy of 0.21) and KPI (accuracy of 0.29) have rather poor results, while the accuracy of $\mathrm{PI}_{4}$ there is very acceptable (accuracy of 0.79 ).

This suggests the following clinical procedure to examine an individual eye. First, one computes GLPI (which has a high performance, close to the KPI's performance in all three groups) as the main diagnose tool. If the value of GLPI suggests a regular cornea, we look at $\mathrm{PI}_{4}$ : if it renders values above the normal threshold of 1 , we classify the patient as a possible keratoconus suspect, requiring further careful examination by the clinician before considering him/her as a candidate for, say, refractive surgery.

\section{Discussion}

The Placido-based anterior corneal topography is an affordable and valuable tool for screening for $\mathrm{KC}^{1}$. Moderate and advanced $\mathrm{KC}$ can be reliably diagnosed by this method, complemented with the biomicroscopic, retinoscopic and pachymetric study ${ }^{1}$. Much more challenging is the detection of this ectatic disorder in its very early or preclinical stages. In the last years, much effort has been devoted to improve the analysis of the corneal topography data in order to increase the ability to diagnose early clinical and subclinical $\mathrm{KC}$ cases. The importance of an early detection of such cases lies in particular in screening out the candidates for the refractive surgery procedures in these weakened and altered corneas. In this sense, a variety of indices or markers have been proposed in the last three decades. The most wellknown and widely used ones are the Rabinowitz and Rabinowitz/McDonnell indices (K, I-S, KISA\%), and the Klyce/Maeda indices (KPI, KCI\%), along with the vertical coma ${ }^{9,13}$, although some others have also been defined ${ }^{33}$. Almost all of them, in accordance with the standard definition of $\mathrm{KC}$, are based on a combination of pachymetry, curvature and corneal power maps obtained by means of corneal topography devices. However, at least in the devices based on Placido disk technology, the corneal power is not the directly measured 
value but a product of a mathematical processing of the raw data, usually obtained under certain a priori assumptions and by proprietary methods, as explained above. This was one of the motivations for the introduction of new corneal irregularity indices ${ }^{36}$ for the Placido disk topographers, defined and analyzed in this work. All of them use exclusively the primary data, that is, the image of the reflection of the mires on the anterior surface of the cornea, bypassing the need to calculate the altimetric or curvature data. It should be stressed that these new indices require only elementary arithmetic manipulation of the digitized images of the mires, and do not intend to imitate the reconstruction of the altimetry or local curvature of the cornea $^{32}$. The aim of the current study was to evaluate in an available sample of normal, keratoconic and preclinical keratoconic eyes these new topographic indices derived directly from the analysis of the digitized images of the Placido rings, and to assess the potential of these indices as a tool for keratoconus detection. We insist that the primary purpose of our markers was not to replace but to complement the standard indices (KPI, KISA\%, and others), eventually providing the clinician with an additional information, especially in the borderline and preclinical KC situations, by detecting an irregular cornea, independently of the type of irregularity it presents.

Regarding the primary corneal indices defined by our research group, statistically significant differences between the control and the $\mathrm{KC}$ groups were found for all indices. Therefore, the primary indices defining different features of the Placido disk images reflected on the cornea were able to discriminate between normal and $\mathrm{KC}$ corneas. A careful observation of the ranges of values of the primary indices in the analyzed groups reveals that there was a relevant area of overlapping for all parameter ranges of both groups. Therefore, these two primary indices showed the best discriminating ability among normal and $\mathrm{KC}$ eyes. $\mathrm{PI}_{2}$ represents a measurement of the dispersion in the location of the centers of the fitted circles to the mires projected on the cornea, considering the diameter of the set of centers as 
well as their drift ${ }^{32}$. Therefore, it characterizes the behavior of the centers of mass of each ring. The new $\mathrm{PI}_{4}$ is an indicator of the global asymmetry of the mires. Specifically, this index measures the slope of the regression line for the centers of the mires. In summary, the direct analysis of the asymmetry of the digitized Placido disks projected on the cornea by means of a corneal topography device allows an effective discrimination between normal and keratoconus corneas.

In the case of the combined index, an excellent discriminating performance of the GLPI (which can be interpreted as a percentage of irregularity) was observed. It was a perfect classifier between keratoconic and normal eyes, and yielded results comparable to the KPI when discriminating between the normal and subclinical KC eyes. Furthermore, a combination of GLPI with $\mathrm{PI}_{4}$ allows achieving an excellent capability of detection of irregular corneas, considering as irregular both the keratoconic and the preclinical keratoconic ones, as Table 6 shows. More specifically, all eyes in the KC group, as well as the majority of the eyes in the preclinical KC group, were classified by this combination of indices as irregular corneas. Thus, the use of the primary corneal indices characterizing the asymmetry of the mires seems to be especially useful for $\mathrm{KC}$ detection, while their combination yields a classification method with excellent discrimination ability between the three groups.

Along with the high sensitivity, another advantages of the corneal indices used in the current study over the standard approaches are (a) their independence from the proprietary algorithms of conversion of the raw ring images into curvature and corneal power, and (b) the mathematical simplicity, with consequent very basic computational requirements. It is convenient to remark that these indices can be easily adapted to any particular commercially available Placido disk topographer; keep in mind that these devices are simple, relatively affordable and easy to use, and represent a vast majority of the topographic devices available in the clinical practice. 
the discrimination between types of pathology but rather a detection of irregularities on the anterior corneal surface. In this sense, we were not trying to replace the standard indices for the detection of KC (such as KPI, I-S or KISA\%).

Currently, studies are being conducted in order to confirm the effectiveness of the defined indices in the detection and characterization of other corneal conditions. The correlation of these indices with higher order corneal aberrations and other optical quality parameters should be also investigated in the future.

In conclusion, the analysis of the digitized images of the Placido disks projected on the cornea is a valid and effective tool for the $\mathrm{KC}$ and preclinical $\mathrm{KC}$ screening that can be used additionally to the existing keratometric criteria. At this stage of our study, we can recommend them as a complementary screening tool designed to alert the clinician, especially in the borderline cases of irregular corneas for which a more exhaustive examination is recommended.

\section{Acknowledgments}

The authors have no proprietary or commercial interest in the medical devices that are involved in this manuscript. This study has been supported in part by the Thematic Network of the Cooperative Sanitary Research (RETIC) RD07/0062 from the Spanish Institute of Health "Carlos III". A.M.-F. and G.CdL are partially supported by the Research Project FIS PI10/01843 from the Spanish Institute of Health "Carlos III". A.M.-F. and D.R.-L. are also supported in part by the research group FQM-229 from Junta de Andalucía and by the project MTM2011-28952-C02-01 from the Ministry of Science and Innovation of Spain and the European Regional Development Fund (ERDF). Additionally, A.M.-F. is partially supported by the Excellence Grant P09-FQM-4643 from Junta de Andalucía. 


\section{References:}

1. Rabinowitz YS. Keratoconus. Surv Ophthalmol 1998; 42:297-319.

2. Piñero DP, Alió JL, Barraquer RI, Michael R, Jiménez R. Corneal biomechanics, refraction, and corneal aberrometry in keratoconus: an integrated study. Invest Ophthalmol Vis Sci 2010; 51: 1948-55.

3. Shah S, Laiquzzaman M, Bhojwani R, Mantry S, Cunliffe I. Assessment of the biomechanical properties of the cornea with the Ocular Response Analyzer in normal and keratoconic eyes. Invest Ophthalmol Vis Sci 2007; 48: 3026-31.

4. Ortiz D, Piñero D, Shabayek MH, Arnalich-Montiel F, Alió JL. Corneal biomechanical properties in normal, post-laser in situ keratomileusis, and keratoconic eyes. J Cataract Refract Surg 2007; 33: 1371-5.

5. Wilson SE and Klyce SD. Quantitative descriptors of corneal topography; a clinical study. Arch of Ophthal; 109: 349-53.

6. Maeda N, Klyce SD, Smolek MK. Comparison of methods for detecting keratoconus using videokeratography. Arch of Ophthal; 103: 870-74

7. Maeda N, Klyce SD, Smolek MK, Thompson HW. Automated keratoconus screening with corneal topography analysis. Invest Ophthalmol Vis Sci; 35: 2749-57.

8. Alió JL, Piñero DP, Alesón A, Teus MA, et al. Keratoconus-integrated characterization considering anterior corneal aberrations, internal astigmatism, and corneal biomechanics. J Cataract Refract Surg. 2011; 37:552-68.

9. Alió JL, Shabayek MH. Corneal higher order aberrations: a method to grade keratoconus. J Refract Surg. 2006 Jun;22(6):539-45. 
10. de Rojas Silva V. Clasificación del Queratocono. In: Queratocono: pautas para su diagnostico y tratamiento. Editor: Albertazzi R. Ediciones Científicas Argentinas para la Keratoconus Society, 2010: 33-97.

11. Li X, Yang H, Rabinowitz YS. Keratoconus: classification scheme based on videokeratography and clinical signs. J Cataract Refract Surg. 2009 Sep;35(9):1597-603.

12. Ambrósio R, Belin MW. Imaging of the Cornea: Topography vs Tomography. J Refract Surg 2010;26(11):847-849.

13. Bühren J, Kühne C, Kohnen T. Defining subclinical keratoconus using corneal firstsurface higher-order aberrations. Am J Ophthalmol 2007;143(3):381-389.

14. Saad A, Gatinel D. Topographic and Tomographic Properties of Forme Fruste Keratoconus Corneas. Invest Ophthalmol Vis Sci 2010;51(11):5546-5555.

15. Saad A, Lteif Y, Azan E, Gatinel D. Biomechanical Properties of Keratoconus Suspect Eyes. Invest Ophthalmol Vis Sci 2010;51(6):29122916.

16. Fontes BM, Ambrósio R, Velarde GC, Nosé W. Ocular Response Analyzer Measurements in Keratoconus with Normal Central Corneal Thickness Compared with Matched Normal Control Eyes. J Refract Surg 2011;27(3):209-215.

17. Piñero DP, Alió JL, Alesón A, Escaf M, Miranda M. Pentacam posterior and anterior corneal aberrations in normal and keratoconic eyes. Clin Exp Optom 2009; 92: 297-303.

18. Gobbe M, Guillon M. Corneal wavefront aberration measurements to detect keratoconus patients. Con Lens Anterior Eye 2005; 28: 57-66. 
19. Barbero S, Marcos S, Merayo-Lloves J, Moreno-Barriuso E. Validation of the estimation of corneal aberrations from videokeratography in keratoconus. $\mathrm{J}$ Refract Surg 2002; 18: 263-70.

20. Carvalho LA. Preliminary results of neural networks and Zernike polynomials for classification of videokeratography maps. Optom Vis Sci 2005; 82: 151-8.

21. Accardo PA, Pensiero S. Neural network-based system for early keratoconus detection from corneal topography. J Biomed Inform 2002; 35: 151-9.

22. Holladay JT. Corneal topography using the Holladay Diagnostic Summary. J Cataract Refract Surg 1997; 23: 209-21.

23. Borderie VM, Laroche L. Measurement of irregular astigmatism using semimeridian data from videokeratographs. J Refract Surg 1996; 12: 595-600.

24. Kalin NS, Maeda N, Klyce SD, Hargrave S, Wilson SE. Automated topographic screening for keratoconus in refractive surgery candidates. CLAO J 1996; 22: 164-7.

25. Rabinowitz YS, McDonnell PJ. Computer-assisted corneal topography in keratoconus. Refract Corneal Surg 1989; 5: 400-8.

26. Dingeldein SA, Klyce SD, Wilson SE. Quantitative descriptors of corneal shape derived from computer-assisted analysis of photokeratographs. Refract Corneal Surg 1989; 5: 372-8.

27. Randleman JB, Russell B, Ward MA, et al. Risk factors and prognosis for corneal ectasia after LASIK. Ophthalmology 2003;110(2):267-275.

28. Binder PS. Analysis of ectasia after laser in situ keratomileusis: risk factors. $\mathbf{J}$ Cataract Refract Surg 2007;33(9):1530-1538. 
29. Prakash, G and Agarwal, A and Mazhari, A I and Kumar, G and Desai, P and Kumar, D A and Jacob, S and Agarwal, A. A new, pachymetry-based approach for diagnostic cutoffs for normal, suspect and keratoconic cornea. Eye 2012: 1-8.

30. Reinstein, D Z and Archer, T J and Gobbe, M, Corneal epithelial thickness profile in the diagnosis of keratoconus, J Refract Surg 25(7) 2009: 604-610.

31. Van Saarloos PP, Constable IJ. Improved method for calculation of corneal topography for any photokeratoscopic geometry. Optom Vis Sci 1991; 68: 957-65.

32. Klein SA. A corneal topography algorithm that produces continuous curvature. Optom Vis Sci 1992; 69: 829-34.

33. Mahmoud AM, Roberts C, Lembach R, Herderick EE, McMahon TT; Clek Study Group. Simulation of machine-specific topographic indices for use across platforms. Optom Vis Sci 2006; 83: 682-93.

34. Greivenkamp JE, Mellinger MD, Snyder RW, Schwiegerling JT, Lowman AE, Miller JM. Comparison of Three Videokeratoscopes in Measurement of Toric Test Surfaces. J Ref Surg 1996; 12: 229-239.

35. Rand RH, Howland HC, Applegate RA. Mathematical model of a Placido disk keratometer and its implications for recovery of corneal topography. Optom Vis Sci 1997; 74: 926-930.

36. Ramos-López D, Martínez-Finkelshtein A, Castro-Luna GM, Piñero D, Alió JL. Placido-based indices of corneal irregularity. Optometry and Vision Science 88 (10) (2011), 1220-1231. 
37. Ahn SJ, Rauh W, Warnecke HJ. Least-Squares Orthogonal Distances Fitting of Circle, Sphere, Ellipse, Hyperbola, and Parabola. Pattern Recognition 2001; 34: 2283-303.

38. Mulchrone KF, Choudhury RK. Fitting an ellipse to an arbitrary shape: implications for strain analysis. Journal of Structural Geology 2004; 26: 14353.

39. Halir R, Flusser J. Numerically stable direct least squares fitting of ellipses, Technical Report. Dept. Software Eng., Charles Univ., Czech Republic 2000. Available at: http://library.utia.cas.cz/prace/980026.ps. Accessed September $17,2012$.

40. Fitzgibbon AW, Pilu M, Fisher RB. Direct least square fitting of ellipses. IEEE Transactions on Pattern Analysis and Machine Intelligence 1999; 21: 476-80.

41. Hart D, Rudman AJ. Least-squares fit of an ellipse to anisotropic polar data: Application to azimuthal resistivity surveys in karst regions. Computers \& Geosciences 1997; 23: 189-94.

42. McCullagh P, Nelder JA. Generalized Linear Models. $2^{\text {nd }}$ edition. Boca Raton: Chapman \& Hall/CRC; 1989.

43. Faraway JF. Linear Models with R. Boca Raton: Chapman \& Hall/CRC; 2005.

44. Bauer DF. Constructing confidence sets using rank statistics. Journal of the American Statistical Association 1972; 67: 687-690.

45. Hollander M, Wolfe DA. Nonparametric Statistical Methods. New York: John Wiley \& Sons; 1973. 
46. Agresti A. Categorical data analysis. Second edition. New York: Wiley; 2001.

47. Fisher RA. Statistical Methods for Research Workers. Oliver \& Boyd; 1970.

48. Picard R, Cook D. Cross-Validation of Regression Models. Journal of the American Statistical Association 1984; 79(387): 575-583.

49. McLachlan GJ, Do KA, Ambroise C. Analyzing microarray gene expression data. Wiley; 2004.

50. Burns DM, Johnston FM, Frazer DG, Patterson C, Jackson AJ. Keratoconus: an analysis of corneal asymmetry. Br J Ophthalmol. 2004; 88(10): 12521255.

51. Smolek MK, Klyce SD. Current keratoconus detection methods compared with a neural network approach, Invest Ophthalmol Vis Sci. 1997; 38(11): 2290-2299. 
Figure 1: An example of a digitized mire (dots) and its approximation by the best-fitcircle (left) and the best-fit-ellipse (right).

Figure 2: Centers $\mathrm{C}_{\mathrm{k}}$ and the corresponding linear fit. Consecutive centers are connected in order to visualize better their relative drift, illustrating the different behaviors captured by indices $\mathrm{PI}_{1}$ (maximum distance) and $\mathrm{PI}_{2}$ (length of the path). 
Table 1 - Mean and standard deviation values for the primary indices in the three groups in the database: Normal (N), Keratoconus (KC) and Keratoconus Suspect (KS).

\begin{tabular}{|c|c|c|c|}
\hline Primary Index & $\begin{array}{c}\text { Normal group } \\
\text { Mean (SD) }\end{array}$ & $\begin{array}{c}\text { KC group } \\
\text { Mean (SD) }\end{array}$ & $\begin{array}{c}\text { KS group } \\
\text { Mean (SD) }\end{array}$ \\
\hline PI $_{\mathbf{1}}$ & $21(16)$ & $194(131)$ & $31(16)$ \\
\hline $\mathbf{P I}_{\mathbf{2}}$ & $28(15)$ & $166(110)$ & $27(13)$ \\
\hline $\mathbf{P I}_{\mathbf{3}}$ & $28(17)$ & $114(90)$ & $17(13)$ \\
\hline $\mathbf{P I}_{\mathbf{4}}$ & $29(23)$ & $208(219)$ & $100(77)$ \\
\hline $\mathbf{A R}(4)$ & $34(9)$ & $55(19)$ & $52(9)$ \\
\hline
\end{tabular}

Table 2 - Summary of the non-parametric tests of equality of means between the three groups. All values in the table are P-values for the Mann-Witney-Wilcoxon test and * meaning that significant differences (level 0.05) in the values of an index between groups were found.

\begin{tabular}{|c|c|c|c|}
\hline Primary Index & N vs KC & KC vs KS & N vs KS \\
\hline $\mathbf{P I}_{1}$ & $<0.01 *$ & $<0.01 *$ & $<0.01 *$ \\
\hline $\mathbf{P I}_{2}$ & $<0.01 *$ & $<0.01 *$ & 0.47 \\
\hline $\mathbf{P I}_{3}$ & $<0.01 *$ & $<0.01 *$ & $<0.01 *$ \\
\hline $\mathbf{P I}_{4}$ & $<0.01 *$ & $<0.01 *$ & $<0.01 *$ \\
\hline $\mathbf{A R}(4)$ & $<0.01 *$ & $<0.01 *$ & 0.49 \\
\hline
\end{tabular}


Table 3 - Value of the area under the ROC curve $\left(A_{z} R O C\right)$ for the indices when classifying between regular eyes (normal group) and irregular eyes (keratoconus group).

\begin{tabular}{|l|l|l|l|l|l|l|}
\hline Index & $\mathbf{P I}_{\mathbf{1}}$ & $\mathbf{P I}_{\mathbf{2}}$ & $\mathbf{P I}_{\mathbf{3}}$ & $\mathbf{P I}_{\mathbf{4}}$ & $\mathbf{A R}(\mathbf{4})$ & GLPI \\
\hline $\mathbf{A}_{\mathbf{z}}$ ROC & 0.987 & 0.989 & 0.880 & 0.936 & 0.837 & 1.000 \\
\hline
\end{tabular}

Table 4 - Accuracy estimates (proportion of individuals well-classified) of the GLPI index defined in (1), for Normal and Keratoconus groups.

\begin{tabular}{|c|c|}
\hline Measurement & Accuracy value \\
\hline 5-fold cross- & 0.95 \\
validation accuracy & \\
\hline 10-fold cross- & \\
validation accuracy & 0.94 \\
estimate & \\
\hline Leave-one-out & \\
cross-validation & \\
accuracy estimate & \\
\hline
\end{tabular}


Table 5 - Joint frequency distributions for KPI and I-S values within the Keratoconus Suspect (KS) group. Each cell contains the number of KS eyes with a value of I-S within the interval at the top of that column and a value of KPI within the interval at the left of that row. Non-shaded cells correspond to those eyes diagnosed as normal eyes by both indices KPI and I-S. Light grey cells are the eyes classified as anomalous by one of these indices, whereas dark grey cells are the eyes screened as abnormal by both of them.

\begin{tabular}{|c|c|c|c|c|}
\hline KPI I-S & {$[-\mathbf{0 . 3}, \mathbf{0 . 4})$} & {$[\mathbf{0 . 4}, \mathbf{1 . 5})$} & {$[\mathbf{1 . 5}, \mathbf{2})$} & {$[\mathbf{2 , 3}]$} \\
\hline$[\mathbf{0 , 5})$ & 2 & 7 & 4 & 0 \\
\hline$[\mathbf{5}, \mathbf{2 3})$ & 0 & 2 & 2 & 1 \\
\hline$[\mathbf{2 3}, \mathbf{4 5})$ & 0 & 2 & 0 & 3 \\
\hline$[\mathbf{4 5}, \mathbf{5 5}]$ & 0 & 0 & 0 & 1 \\
\hline
\end{tabular}

Table 6 - Summary of results of classification ability of some of the proposed indices and the KPI. All the values within the table are the accuracy of each index when classifying in the stated group.

\begin{tabular}{|l|l|l|l|}
\hline Index & Normal & KC & KC Suspects \\
\hline GLPI & 1.00 & 1.00 & 0.21 \\
\hline KPI & 1.00 & 1.00 & 0.25 \\
\hline PI $_{4}$ & 0.87 & 0.90 & 0.79 \\
\hline
\end{tabular}


Click here to download high resolution image

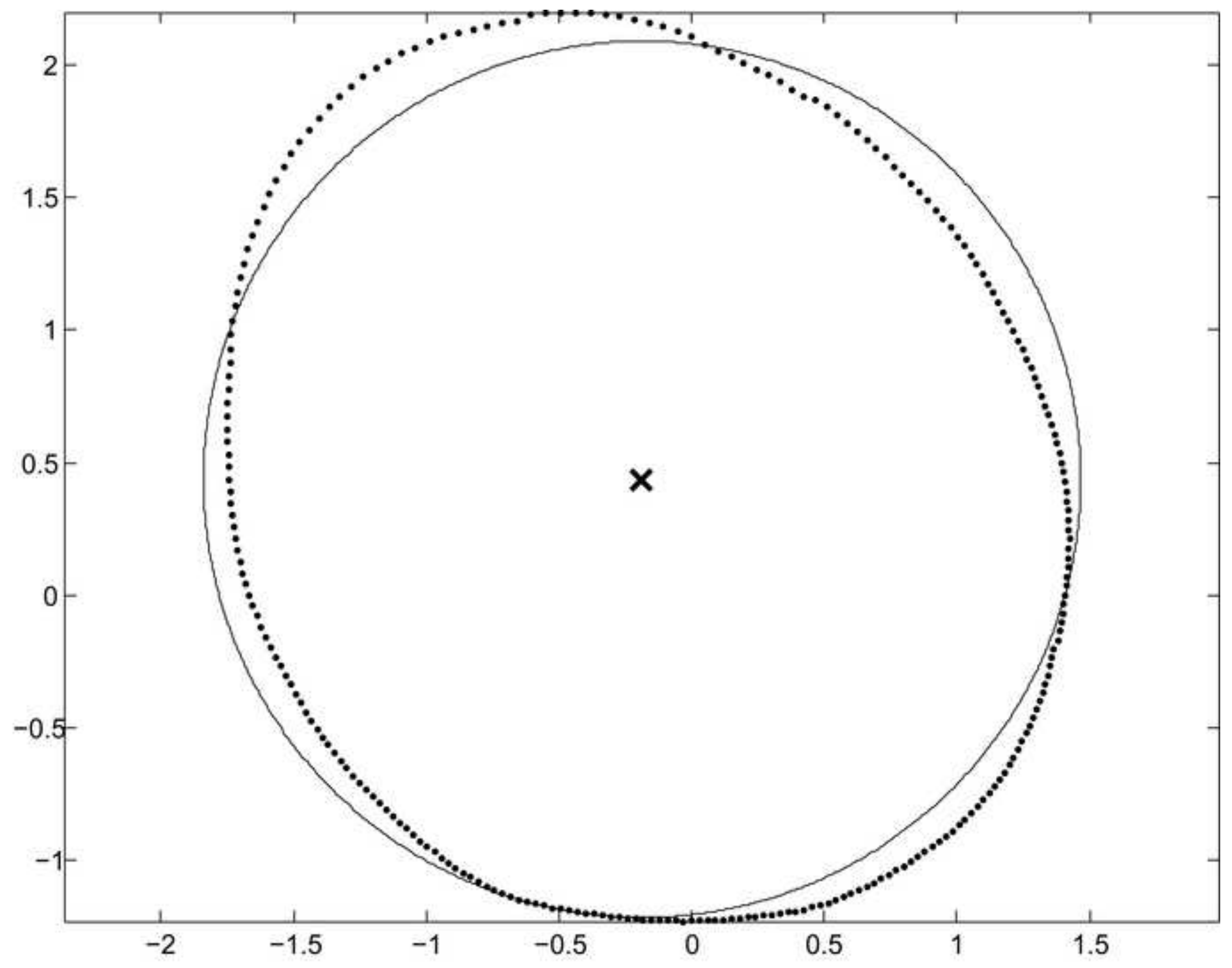


Click here to download high resolution image

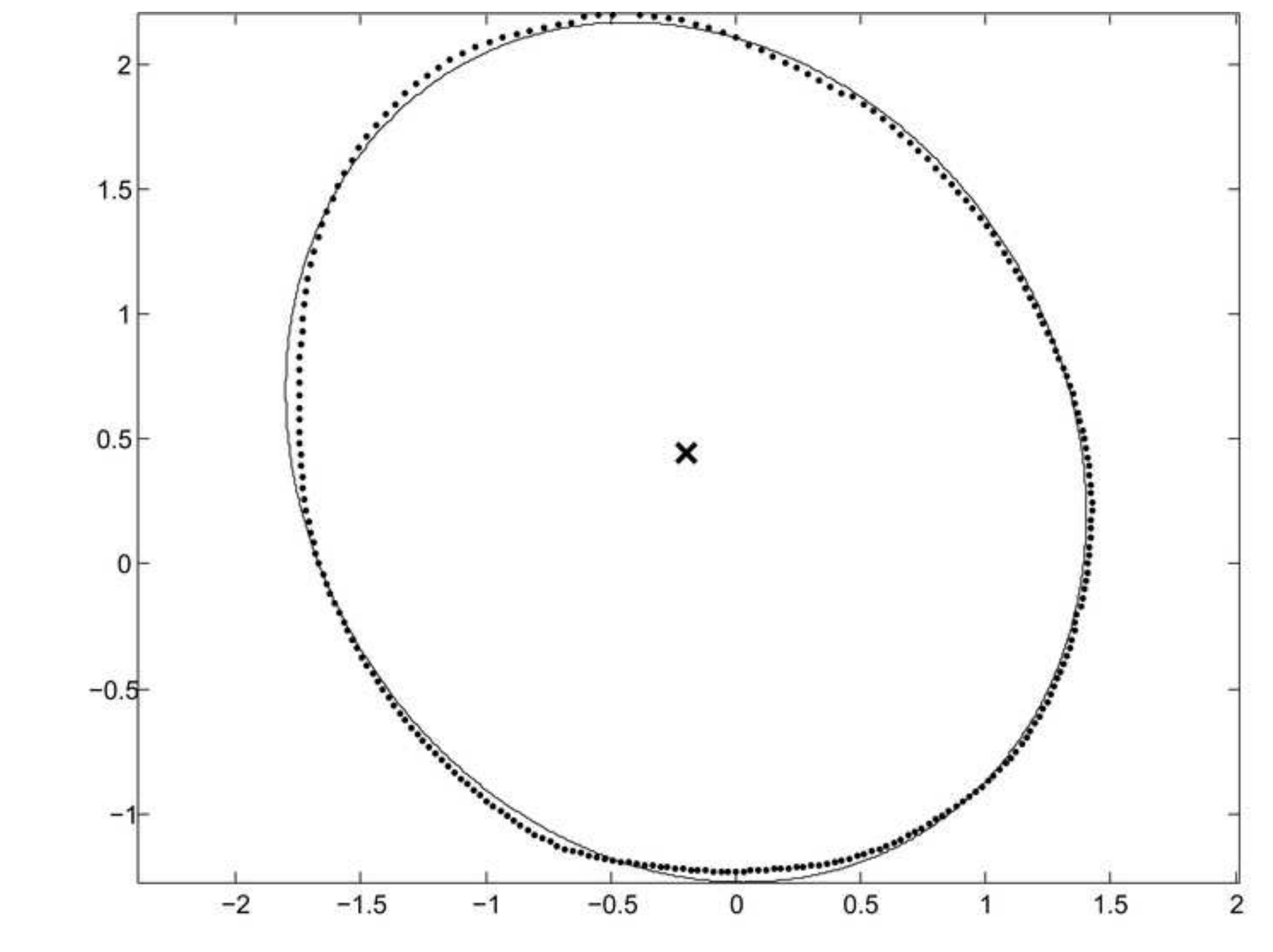




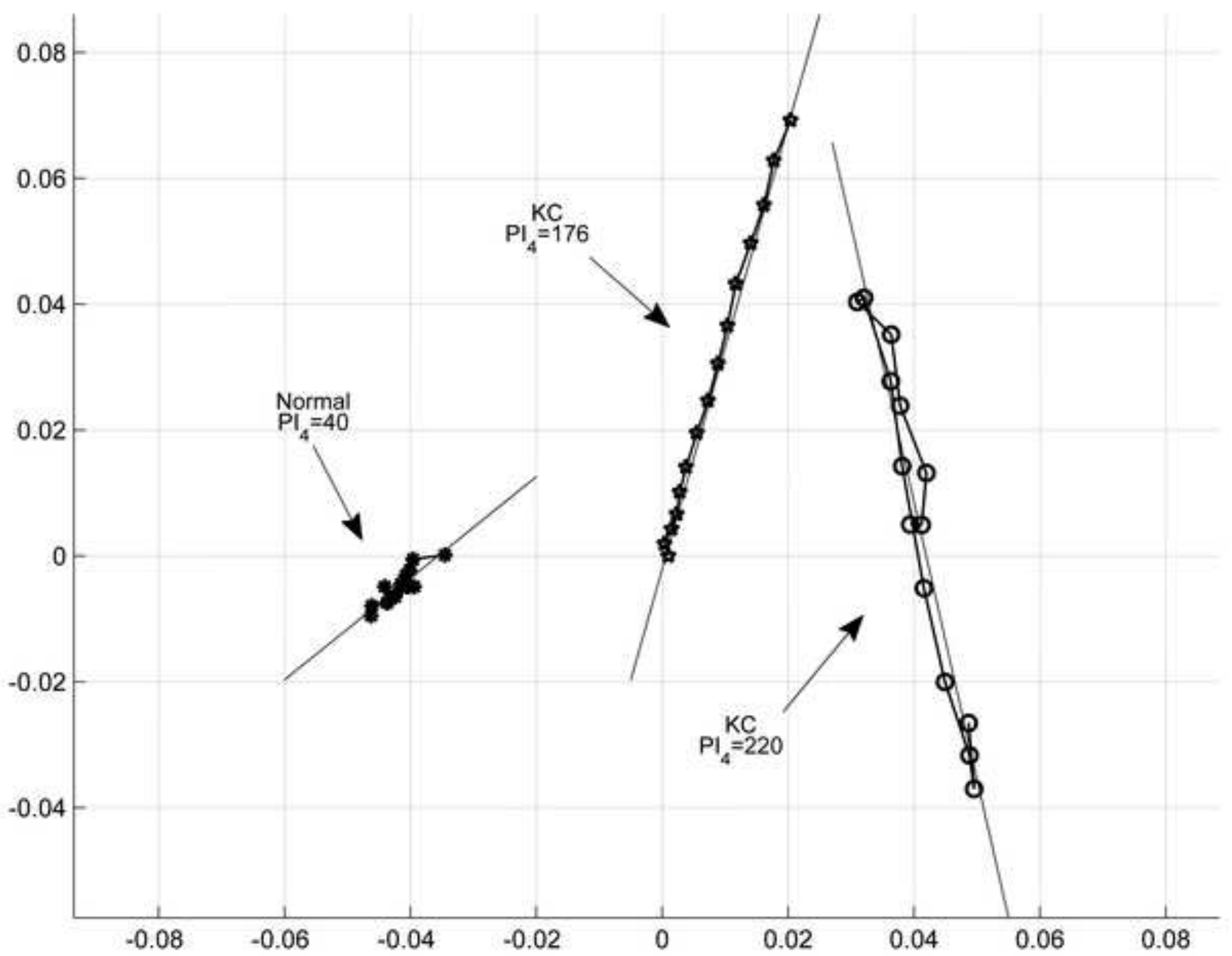




\section{Figure}

Click here to download high resolution image

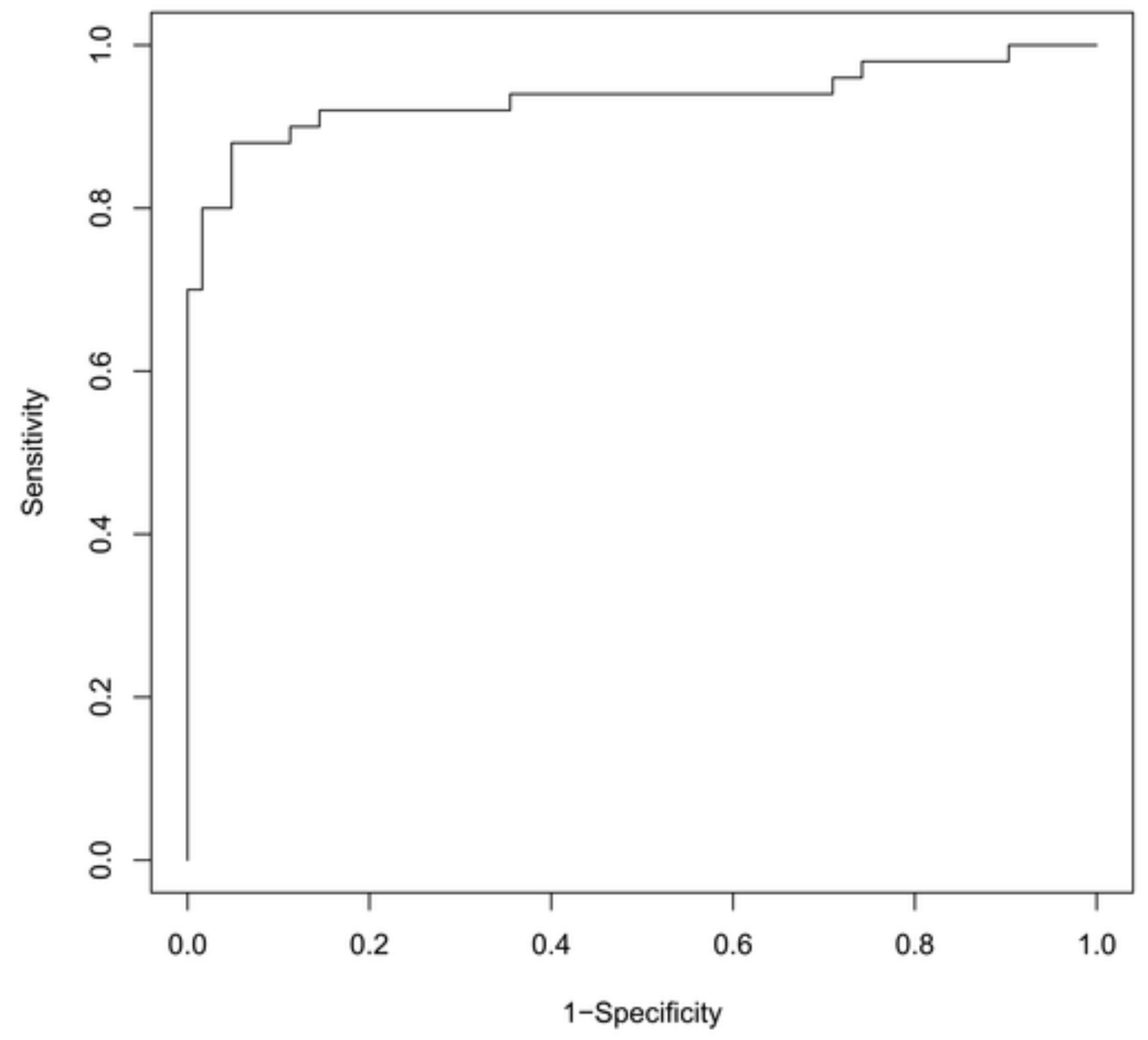




\section{Figure}

Click here to download high resolution image

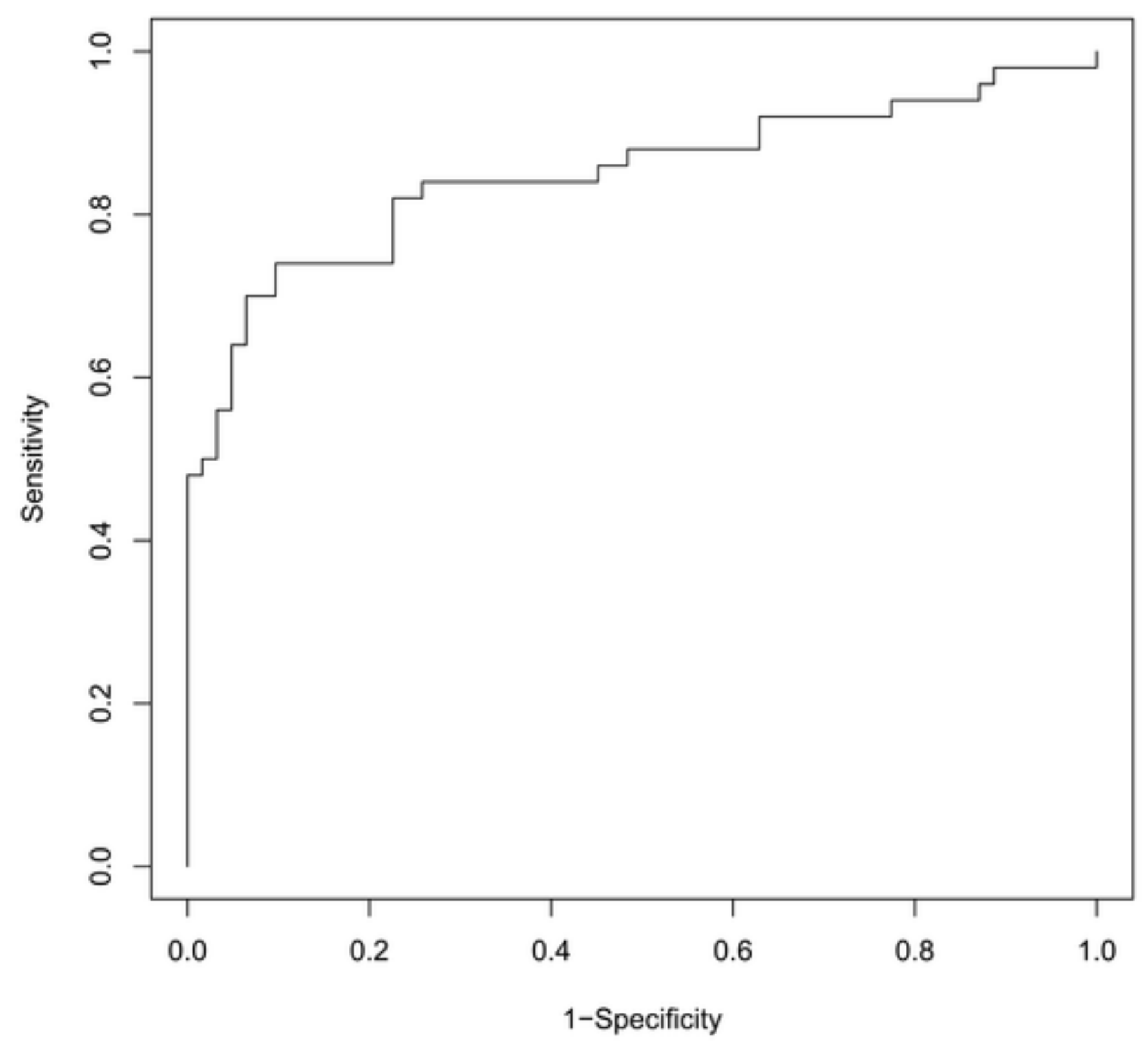

